\title{
DETERMINAÇÃO DOS TEORES DE COBRE EM DIFERENTES EXTRATOS DE HORTALIÇAS DO TIPO A E B ${ }^{1}$
}

\author{
Édira Castello Branco de ANDRADE ${ }^{2 *}$, Anderson Junger TEODORO²,
}

\author{
Iracema TAKASE ${ }^{3}$
}

\begin{abstract}
RESUMO
Amostras de hortaliças dos tipos A e B foram analisadas quanto ao teor total de cobre através de espectroscopia de absorção atômica em chama, e o método de extração seqüencial foi aplicado com os seguintes extratores: solução de CaCl ${ }_{2} 1,0 \mathrm{M}$; solução de ácido acético 0,1M; solução de ácido acético 0,5M / acetato de amônio 5\% [pH=5,0]; solução de $\mathrm{NaOH} 0,1 \mathrm{M}$ e solução de $\mathrm{HCl} 0,5 \mathrm{M}$. Na análise da rejeição de resultados foi aplicado o teste de Grubbs e na comparação dos dados o teste t de Student foi utilizado. Em média $25,4 \%$ do teor total de cobre foram extraídos com solução de $\mathrm{CaCl}_{2} 1,0 \mathrm{M}$, sendo o máximo de $50,5 \%$ e o mínimo de $8,3 \%$. Em média $14,8 \%$ do teor total de cobre, sendo que $32,6 \%$ e 6,4\%, máximo e mínimo respectivamente, foram extraídos com solução de ácido acético $0,1 \mathrm{M}$. Com relação ao uso de solução de ácido acético $0,5 \mathrm{M} /$ acetato de amônio $5 \%$ [pH=5,0], o mínimo extraído obteve valor de $6,2 \%$ e o máximo $27,6 \%$, com média de $13,7 \%$. O extrator de $\mathrm{NaOH} 0,1 \mathrm{M}$ foi o que teve menor extração, cerca de $10,5 \%$ e com extrator de $\mathrm{HCl} 0,5 \mathrm{M}$ a extração foi em média de $12,6 \%$. A fração extraída de cobre nas amostras foi de no mínimo 34,5\% e no máximo $100 \%$. Observa-se que para a maioria das amostras, o cobre se encontra sob a forma de, no mínimo, 6 espécies químicas distintas.

Palavras-chave: cobre; extração seqüencial; biodisponibilidade.
\end{abstract}

\section{SUMMARY}

DETERMINATION OF COPPER IN DIFFERENTS EXTRACTS IN SAMPLES OF A AND B VEGETABLES. Total copper content analysed in samples of $\mathrm{A}$ and $\mathrm{B}$ vegetables through atomic flame absorption spectroscopy and sequential extraction were performed by using the following extractants: $\mathrm{CaCl}_{2} 1.0 \mathrm{M}$ solution; acetic acid $0.1 \mathrm{M}$; acetic acic $0.5 \mathrm{M} /$ ammonium acetate $5 \%$ solution [pH $\left.=5.0\right]$; $\mathrm{NaOH} 0.1 \mathrm{M}$ solution and $\mathrm{HCl} 0.5 \mathrm{M}$ solution. For evaluation of the results Grubbs test and Student test were used. On average $25.4 \%$ of total copper were extracted in $\mathrm{CaCl}_{2}$ 1.0M solution with a maximum extraction of $50.5 \%$ and a minimum of $8.3 \%$. On average $14.8 \%$ of the total amount of copper, a maximum of $32.6 \%$ and a minimum of $64 \%$ were extracted in acetic acid $0.1 \mathrm{M}$ solution. Using acetic acid $0.5 \mathrm{M} /$ ammonum acetate $5 \%[\mathrm{pH}=5.0]$ solution, the minimum extracted was $6.2 \%$ and the maximum $27.6 \%$ with $13.75 \%$ as average. The less effective extractant was $\mathrm{NaOH} 0.1 \mathrm{M}(10.5 \%)$ and $\mathrm{HCl} 0.5 \mathrm{M}$ extractant which had anaverage of $12.6 \%$ of extraction. The copper extracted was present at least in of six different chemical species.

Keywords: copper; sequential extraction; bioavailability.

\section{1 - INTRODUÇÃO}

Os minerais constituem um grupo de elementos largamente distribuídos na natureza e que exercem papel dos mais importantes em diversas funções e setores do organismo. Eles atuam tanto na forma iônica quanto como constituintes de compostos (enzimas, hormônios, secreções e proteínas do tecido orgânico) $[9,26]$.

Dentre os vários minerais existentes destaca-se o cobre, classificado como microelemento essencial ou elemento-traço e que exerce funções essenciais ao organismo [26].

O cobre é um microelemento de número atômico 29 e peso atômico de 63,55 e essencial para diversas funções como: mobilização do ferro para a sintese de hemoglobina e componente de várias enzimas, como citocromo C-oxidase, superóxido dismutase, monoamino-oxidase, etc... [9, 26]. O cobre é absorvido na proporção de 40 a

\footnotetext{
Recebido para publicação em 18/06/2003. Aceito para publicação em 12/05/2004 (001153).

2. Departamento de Tecnologia dos Alimentos, Escola de Nutrição, UNIRIO, UNIVERSIDADE DO RIO DE JANEIRO, Rua Xavier Sigaud n $n^{\circ} 290$, prédio II, $3^{\circ}$ andar, Urca, Rio de janeiro, CEP 22290-180, Email: ediracba@unirio.br

3. Departamento de Química Analítica, UFRJ

* A quem a correspondência deve ser enviada.
}

50\% da ingestão oral, no estômago e duodeno proximal por transporte ativo junto a aminoácidos ou por difusão. Uma vez absorvido é transportado para o fígado, ligado à albumina e transcupreína, incorporando-se então a ceruloplasmina e várias metaloenzimas, que permite o transporte do cobre para os tecidos extra-hepáticos [9].

A excreção é feita via fecal e, em maior teor pela bile, assim como a urina e o suor. Sua deficiência provoca anemia, leucopenia, neutropenia, hiperucemia, retardo no crescimento; enquanto que sua toxicidade provoca diarréia, náusea, vômitos, cirrose, anemia e bronquite [9, 26].

Existem várias interações entre vitaminas e minerais e o conhecimento destas, permite um maior controle de algumas variáveis, para uma melhor utilização do nutriente. As interações físico-químicas entre nutrientes incluem: adsorção e formação de complexos e precipitação, com influência da estabilidade de ambos, interferindo na biodisponibilidade dos metais $[7,8,20]$.

A biodisponibilidade do cobre está comprometida com o excesso de vitamina $\mathrm{C}$ e do zinco, ambos diminuem o processo de absorção do cobre [6, 7, 9, 26].

Vale ressaltar que em relação à forma química dos elementos traço, a mais disponivel é a forma de compostos orgânicos em relação aos sais [10, 25].

Através da determinação do teor total do metal ingerido, não é possível medir o quanto deste metal será absorvido. Entretanto através da técnica de especiação 
dos metais, que é definida como a determinação da concentração das formas físico-químicas individuais dos elementos que, em conjunto, constitua a concentração total do mesmo na amostra, podemos ter novos dados para prever sua absorção [10, 19, 25].

Para a especiação em amostras sólidas, inicialmente deve ser aplicado um método de extração seqüencial ou seletivo. Um elemento pode estar presente em um sólido sob a forma de espécies adsorvidas na superfície, co-precipitadas, ocluidos ou compostos de coordenação. A maioria dos esquemas de extração seqüencial inclui diferentes grupos de extratores, já que ela se baseia na progressão de extratores fracos, como a água, até um extrator forte, como um ácido concentrado [10, $12,19,25]$.

Os primeiros extratores da extração seqüencial geralmente são aqueles com propriedades de troca iônica, deslocando íons de sítios iônicos específicos, sendo representados por soluções iônicas, como o cloreto de magnésio e de cálcio e nitrato de sódio. Em seguida, são utilizados extratores com propriedades de dissolução de carbonatos, já que metais ligados a carbonatos são sensíveis à variação de $\mathrm{pH}$. Estes são representados por soluções tampão de ácido/acetato de amônia $\mathrm{pH}=5,0$. Os extratores com propriedades de redução ácida continuam o processo de extração e promovem a dissolução de oxihidratos de ferro e manganês, tendo como exemplo a solução diluída de ácido acético. Por fim, dois últimos tipos de extratores são utilizados: primeiro aqueles que enfraquecem a ligação do metal com compostos orgânicos e sulfetos, representados por soluções ácidas de peróxido de hidrogênio, soluções de hidróxido de sódio $\mathrm{pH}=12,0$ e ácido clorídrico $\mathrm{pH}=2,0$, pois permitem a degradação da matéria orgânica, por hidrólise ou oxidação, facilitando a liberação dos metais; e, por último são utilizados os extratores que promovem a dissolução dos silicatos e minerais, representados por ácidos fortes. Um método de especiação ideal é definido como aquele que pode fornecer informações desejáveis, sem alterar a amostra original em momento algum [11, 12, 17, 18, 19, 21, 22, 23, 24, 25].

As hortaliças podem ser classificadas segundo o teor de glicídios onde as do tipo A contém até $5 \%$ de glicídios, as do tipo B 10\% e as do tipo C até 20\% do teor em glicídios [16].

Este trabalho tem como objetivo promover a extração seqüencial e análise de cobre em hortaliças do tipo A e B.

\section{2 - MATERIAL E MÉTODOS}

\section{1 - Amostras}

As amostras analisadas foram: hortaliças do tipo A abobrinha-verde, agrião, bertalha, brócolis, chicória, espinafre e tomate; hortaliças do tipo B -abóbora, abóbora-moranga, beterraba, cebola, cenoura, couve, couve-flor, ervilha, jiló, nabo, pimentão, quiabo, repolho e vagem.

\section{2 - Tratamento das amostras}

As amostras foram fracionadas e parte retirada para determinação da umidade através do processo de perda de substâncias voláteis em estufa a $105^{\circ} \mathrm{C}$, sendo o restante dessecado na mesma temperatura até total secagem. As amostras secas após homogeneização foram calcinadas a $550^{\circ} \mathrm{C}$ por um período mínimo de 2 horas. As cinzas foram dissolvidas em $\mathrm{HCl}$ 2,0M e transferidas quantitativamente para balão volumétrico de $25 \mathrm{~mL}$ [5].

\section{3 - Extração seqüencial}

Foram utilizados cerca de $5 \mathrm{~g}$ de amostra seca e cada amostra passou por processo de extração seqüencial. Os extratores utilizados foram: solução de $\mathrm{CaCl}_{2}$ 1,0M; solução de ácido acético $0,1 \mathrm{M}$; solução de ácido acético 0,5M / acetato de amônio 5\% [pH=5,0]; solução de $\mathrm{NaOH} \mathrm{0,1M} \mathrm{e}$ solução de $\mathrm{HCl}$ 0,5M. Cada extrator esteve em contato com a amostra durante 1 hora, sendo depois filtrado, e este utilizado para determinação do teor total de cobre.

- Determinação do teor total de cobre - o teor total de cobre nas amostras foi determinado através da espectrometria de absorção atômica em chama [5].

- Tratamento estatístico - na análise da rejeição de resultados foi aplicado o teste de Grubs e o teste t de Student foi usado [14].

- Garantia da qualidade - os processos de extração seqüencial, determinação de umidade e de determinação dos teores totais de cobre foram feitos em triplicata. O aparelho de absorção atômica foi sempre previamente calibrado com solução analítica do respectivo metal.

\section{3 - RESULTADOS E DISCUSSÃO}

Os resultados obtidos são apresentados a seguir. Na Tabela 1 encontram-se os teores de umidade e de cobre nas amostras analisadas. Os dados foram tratados estatisticamente aplicando o teste de Grubs, nível de confiança 95\%, não houve dados rejeitados. Em média, as hortaliças do tipo A apresentaram 0,09mg\% de cobre e as hortaliças do tipo B 0,08mg\%. Comparando esses valores com os obtidos por ANDRADE et al. [1], na análise destes metais em frutas; em hortaliças do tipo C [2]; em cereais crus e processados [3]; e em leguminosas cruas e processadas [4], constata-se que em relação ao cobre, as hortaliças A e B possuem um teor total médio menor do que frutas, cereais, hortaliças C e leguminosas, já que estes apresentam teores médios de cobre de 0,13mg\%, 0,21 $\mathrm{mg} \%, 0,24 \mathrm{mg} \%$ e $0,89 \mathrm{mg} \%$, respectivamente.

Estima-se com a "Ingestão Dietética Diária Considerada Segura e Adequada" (ESADDI - Estimate and Adequate Daily Dietary Intake) para o cobre 1,5 a 3,0mg/ dia $[13,15]$. Considerando que o teor médio de cobre nas amostras de hortaliças dos tipos A e B foi de $0,085 \mathrm{mg} \%$, $100 \mathrm{~g}$ destes alimentos que corresponde a porção usualmente ingerida dos mesmos, pode fornecer cerca de 5,7\% do valor da RDA. 
A Tabela 2 apresenta os teores de cobre extraídos pelo método de extração seqüencial. Os dados foram tratados estatisticamente aplicando o teste de Grubs, nível de confiança $95 \%$, não foi rejeitado nenhum resultado. A fração correspondente ao extrator I representa a fração com propriedades de troca iônica, deslocando sítios iônicos específicos, ou seja, é uma fração facilmente solúvel [19, 22, 25]. Em média 25,4\% foram extraídos do total de cobre encontrado nas hortaliças, sendo o máximo de 50,5\% e o mínimo de $8,3 \%$. Vale ressaltar as amostras de couve-flor e bertalha que apresentaram extração de 50\% do teor total de cobre.

No caso do extrator II, que possui propriedades de redução ácida e promove dissolução dos oxihidratos de ferro e manganês $[11,18]$, a fração extraída possui uma solubilidade média de $14,8 \%$, sendo $32,6 \%$ e $6,4 \%$, o máximo e o mínimo extraídos, respectivamente. Observa-se que as amostras de abóbora, abobrinha-verde, agrião, bertalha, brócolis, chicória, couve-flor, espinafre, jiló, nabo, pimentão, repolho e tomate, apresentaram cerca de $50 \%$ da extração pelo extrator I. Aplicandose o teste $\mathrm{t}$ de Student, intervalo de confiança 95\% observa-se que as amostras de abóbora-moranga, beterraba, cebola, cenoura, couve, ervilha, quiabo e vagem tiveram o cobre extraído com o extrator II, em igual proporção do extrator I.

Com relação ao extrator III, a fração extraída corresponde a fração carbonatada que sofre alta variação de $\mathrm{pH}[11,18]$. O mínimo extraído foi de $6,2 \%$ e o máximo $27,6 \%$ com média de $13,7 \%$. Observa-se que com exceção das amostras de bertalha, cenoura, couve-flor, jiló e vagem, as demais apresentaram teor de cobre extraído similar ao teor extraído com o extrator II, ao aplicar o teste t de Student, intervalo de confiança 95\%.

TABELA 1. Teor $\%$ de umidade e teor total de cobre $\mathrm{mg} \%$ em hortaliças dos tipos A e B

\begin{tabular}{lcc}
\hline \multicolumn{1}{c}{ Alimentos } & Umidade $\%$ & Cobre $\mathrm{mcg} / \mathrm{g}$ \\
\hline Abóbora & $96,00 \pm 0,00$ & $0,48 \pm 0,08$ \\
Abóbora-Moranga & $89,33 \pm 1,15$ & $0,61 \pm 0,04$ \\
Abobrinha-verde & $95,33 \pm 1,15$ & $0,68 \pm 0,02$ \\
Agrião & $90,67 \pm 1,15$ & $1,13 \pm 0,15$ \\
Bertalha & $90,00 \pm 0,00$ & $0,91 \pm 0,04$ \\
Beterraba & $90,00 \pm 0,00$ & $0,81 \pm 0,01$ \\
Brócolis & $88,66 \pm 1,15$ & $1,00 \pm 0,00$ \\
Cebola & $89,33 \pm 1,15$ & $0,49 \pm 0,01$ \\
Cenoura & $89,33 \pm 1,15$ & $0,98 \pm 0,08$ \\
Couve & $86,67 \pm 1,15$ & $1,20 \pm 0,10$ \\
Couve-flor & $90,00 \pm 0,00$ & $0,46 \pm 0,01$ \\
Chicória & $96,98 \pm 1,15$ & $0,56 \pm 0,07$ \\
Ervilha & $88,00 \pm 12,0$ & $0,58 \pm 0,02$ \\
Espinafre & $94,67 \pm 1,15$ & $1,33 \pm 0,30$ \\
Jiló & $92,00 \pm 0,00$ & $1,30 \pm 0,10$ \\
Nabo & $92,00 \pm 0,00$ & $0,65 \pm 0,02$ \\
Pimentão & $94,00 \pm 0,00$ & $0,61 \pm 0,00$ \\
Quiabo & $90,67 \pm 1,15$ & $1,10 \pm 0,10$ \\
Repolho & $92,67 \pm 1,15$ & $0,87 \pm 0,12$ \\
Tomate & $94,00 \pm 1,15$ & $1,07 \pm 1,13$ \\
Vagem & $92,67 \pm 1,15$ & $0,73 \pm 0,06$ \\
\hline
\end{tabular}

As frações extraídas pelos extratores IV e V, que enfraquecem ligações do metal com compostos orgânicos $[11,18]$, representaram em média 10,5\% e 12,6\% do teor total de cobre das amostras. Aplicando o teste t de Student, intervalo de confiança 95\%, observa-se que exceto as amostras de abóbora, abóbora-moranga, agrião, bertalha, couve-flor, couve, ervilha, jiló e vagem as demais amostras apresentaram teor similar de cobre extraído com os extratores IV e V.

TABELA 2. Teores de cobre em mcg/g nos extratos de hortaliças do tipo A e B

\begin{tabular}{|c|c|c|c|c|c|}
\hline \multirow{2}{*}{ Amostras } & \multicolumn{5}{|c|}{ Extratores } \\
\hline & I & II & III & IV & V \\
\hline Abóbora & $0,15 \pm 0,01$ & $0,07 \pm 0,01$ & $0,05 \pm 0,01$ & $0,03 \pm 0,00$ & $0,09 \pm 0,03$ \\
\hline Abóbora-Moranga & $0,12 \pm 0,01$ & $0,10 \pm 0,01$ & $0,10 \pm 0,01$ & $0,07 \pm 0,01$ & $0,12 \pm 0,01$ \\
\hline Abobrinha-verde & $0,13 \pm 0,01$ & $0,05 \pm 0,01$ & $0,05 \pm 0,01$ & $0,07 \pm 0,01$ & $0,06 \pm 0,00$ \\
\hline Agrião & $0,31 \pm 0,01$ & $0,12 \pm 0,01$ & $0,11 \pm 0,05$ & $0,06 \pm 0,00$ & $0,10 \pm 0,01$ \\
\hline Bertalha & $0,46 \pm 0,03$ & $0,20 \pm 0,05$ & $0,14 \pm 0,03$ & $0,10 \pm 0,01$ & $0,14 \pm 0,01$ \\
\hline Beterraba & $0,10 \pm 0,02$ & $0,10 \pm 0,02$ & $0,09 \pm 0,02$ & $0,05 \pm 0,01$ & $0,07 \pm 0,01$ \\
\hline Brócolis & $0,28 \pm 0,01$ & $0,11 \pm 0,03$ & $0,13 \pm 0,02$ & $0,09 \pm 0,01$ & $0,09 \pm 0,00$ \\
\hline Cebola & $0,14 \pm 0,03$ & $0,16 \pm 0,02$ & $0,11 \pm 0,03$ & $0,10 \pm 0,01$ & $0,10 \pm 0,03$ \\
\hline Cenoura & $0,29 \pm 0,00$ & $0,27 \pm 0,01$ & $0,13 \pm 0,00$ & $0,10 \pm 0,00$ & $0,12 \pm 0,11$ \\
\hline Chicória & $0,12 \pm 0,01$ & $0,06 \pm 0,02$ & $0,07 \pm 0,02$ & $0,04 \pm 0,01$ & $0,04 \pm 0,01$ \\
\hline Couve & $0,37 \pm 0,03$ & $0,25 \pm 0,05$ & $0,26 \pm 0,03$ & $0,19 \pm 0,06$ & $0,11 \pm 0,02$ \\
\hline Couve-flor & $0,23 \pm 0,00$ & $0,10 \pm 0,03$ & $0,06 \pm 0,01$ & $0,05 \pm 0,01$ & $0,10 \pm 0,00$ \\
\hline Ervilha & $0,15 \pm 0,02$ & $0,16 \pm 0,03$ & $0,16 \pm 0,03$ & $0,11 \pm 0,03$ & $0,15 \pm 0,04$ \\
\hline Espinafre & $0,29 \pm 0,01$ & $0,11 \pm 0,00$ & $0,08 \pm 0,02$ & $0,08 \pm 0,04$ & $0,09 \pm 0,02$ \\
\hline Jiló & $0,43 \pm 0,04$ & $0,19 \pm 0,06$ & $0,15 \pm 0,01$ & $0,14 \pm 0,06$ & $0,21 \pm 0,07$ \\
\hline Nabo & $0,12 \pm 0,01$ & $0,05 \pm 0,00$ & $0,09 \pm 0,02$ & $0,06 \pm 0,01$ & $0,08 \pm 0,00$ \\
\hline Pimentão & $0,11 \pm 0,02$ & $0,07 \pm 0,02$ & $0,09 \pm 0,01$ & $0,06 \pm 0,01$ & $0,07 \pm 0,02$ \\
\hline Quiabo & $0,09 \pm 0,01$ & $0,07 \pm 0,00$ & $0,09 \pm 0,03$ & $0,06 \pm 0,01$ & $0,07 \pm 0,01$ \\
\hline Repolho & $0,19 \pm 0,01$ & $0,08 \pm 0,02$ & $0,06 \pm 0,01$ & $0,06 \pm 0,01$ & $0,06 \pm 0,00$ \\
\hline Tomate & $0,29 \pm 0,01$ & $0,14 \pm 0,01$ & $0,12 \pm 0,01$ & $0,10 \pm 0,03$ & $0,11 \pm 0,00$ \\
\hline Vagem & $0,12 \pm 0,03$ & $0,10 \pm 0,00$ & $0,15 \pm 0,01$ & $0,10 \pm 0,02$ & $0,06 \pm 0,02$ \\
\hline
\end{tabular}

Extrator I - Solução de cloreto de cálcio - 1.00M pH 7:

Extrator II - Solução ácido acético - 0,10M com Acetato de amônio - 5,00\% pH 5,00 ;

Extrator III - Solucão de ácido acético $-1,00 \mathrm{M}$

Extrator IV - Solução hidróxido de sódio - 0,10M;

Extrator V - Solução de ácido clorídrico - 0,50M

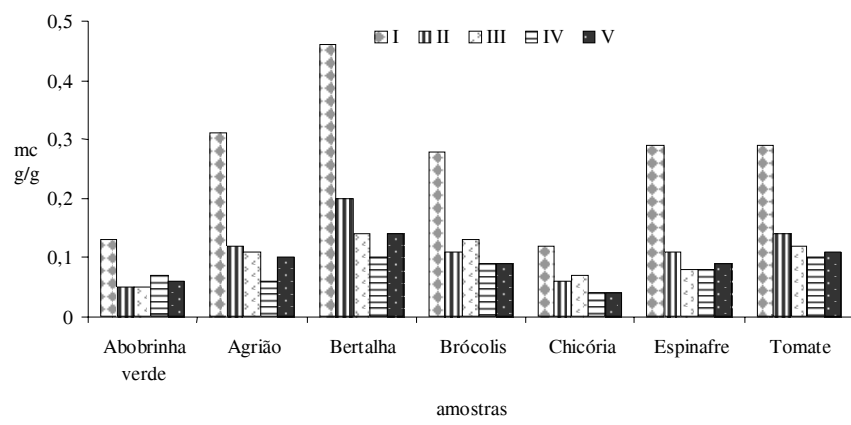

Extrator I - Solução de cloreto de cálcio - 1,00M pH 7;
Extrator II - Solução ácido acético - 0,10M com Acetato de amônio - 5,00\% pH 5xtrato

Extrator III - Solução de ácido acético -1,00M:

Extrator IV - Solução hidróxido de sódio - 0,10M:

Extrator V - Solução de ácido clorídrico - 0,50M

FIGURA 1. Extração sequencial de cobre em $\mathrm{mcg} / \mathrm{g}$ de hortaliças do tipo 
A Figura 1 apresenta os teores de cobre extraídos durante o processo de extração seqüencial em hortaliças do tipo A. Observa-se que todas as amostras apresentaram maior extração com o extrator I, e o comportamento frente aos diferentes extratores foi similar.

A Figura 2 mostra os teores de cobre extraídos pelo processo de extração seqüencial em hortaliças do tipo B. Comparando estas amostras com as hortaliças do tipo A verifica-se que o comportamento frente aos diferentes extratores é bem distinto. Considerando que a maioria das amostras apresenta, no mínimo, seis espécies químicas distintas de cobre, as extraídas pelos extratores I, II, III, IV e V e a fração não extraída, acredita-se que para avaliar a biodisponibilidade deste metal nestas amostras o estudo deva ser para cada amostra específica, em função dos diferentes comportamentos frente aos extratores.

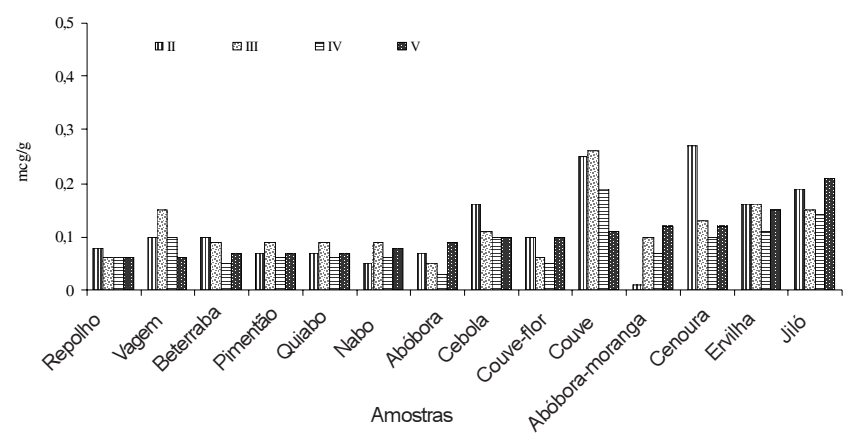

Extrator I - Solução de cloreto de cálcio - 1,00M pH 7;

Extrator II - Solução ácido acético - 0,10M com Acetato de amônio - 5,00\% pH 5,00: Extrator III - Solução de ácido acético-1,00M;

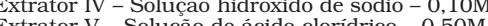

FIGURA 2. Extração seqüencial de cobre em mcg/g de hortaliças do tipo B

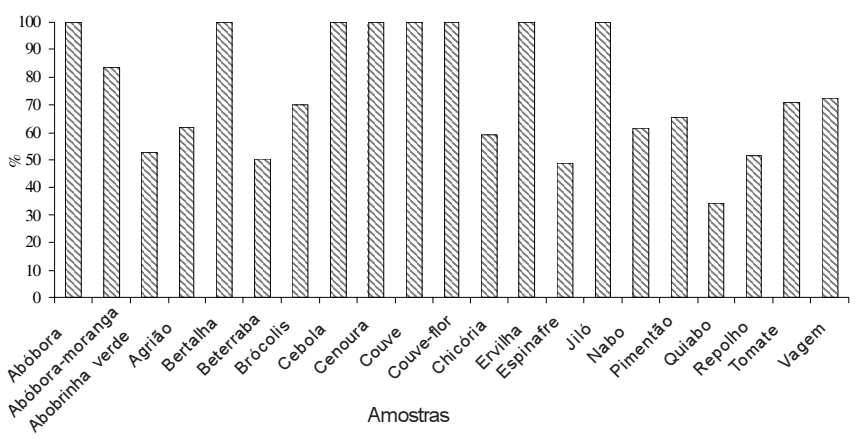

FIGURA 3. Teor \% de cobre extraído de hortaliças do tipo A e B através de extração seqüencial

A Tabela 3 mostra os teores totais de cobre nas amostras in natura e na fração total obtida pelo somatório dos teores de cobre extraídos durante o processo de extração seqüencial. Aplicando o teste t de student, intervalo de confiança 95\%, observa-se que 8 amostras apresentaram extração total do teor de cobre. As amostras de brócolis, tomate e vagem tiveram extração superior a $70 \%$ do teor total de cobre. Abobrinha-verde, agrião, beterraba, chicória, nabo, pimentão e repolho apresentaram extração superior a $50 \%$ do teor total de cobre. Quiabo e espinafre apresentaram extração superior a 35\% do teor de cobre. A Figura 3 apresenta o percentual de cobre total extraído das amostras. Observa-se que a extração de cobre das amostras de abóbora, bertalha, cebola, cenoura, couve, couve-flor, ervilha e jiló foi considerada $100 \%$ após os dados terem sido tratados estatisticamente com teste t de student, intervalo de confiança 95\%.

TABELA 3. Teor em mg\% de cobre nas amostras in natura e na fração total extraída

\begin{tabular}{lcc}
\multicolumn{1}{c}{ Amostras } & $\begin{array}{c}\mathrm{mcg} / \mathrm{g} \mathrm{Cu} \text { amostras } \\
\text { in natura }\end{array}$ & $\begin{array}{c}\text { Total de Cu extraído } \\
\text { em mcg/g }\end{array}$ \\
Abóbora & $0,48 \pm 0,08$ & 0,39 \\
Abóbora-moranga & $0,61 \pm 0,04$ & 0,51 \\
Abobrinha-verde & $0,68 \pm 0,02$ & 0,36 \\
Agrião & $1,13 \pm 0,15$ & 0,70 \\
Bertalha & $0,91 \pm 0,04$ & 1,04 \\
Beterraba & $0,81 \pm 0,01$ & 0,41 \\
Brócolis & $1,00 \pm 0,00$ & 0,70 \\
Cebola & $0,49 \pm 0,01$ & 0,61 \\
Cenoura & $0,98 \pm 0,08$ & 0,91 \\
Couve & $1,20 \pm 0,10$ & 1,18 \\
Couve-flor & $0,46 \pm 0,01$ & 0,54 \\
Chicória & $0,56 \pm 0,07$ & 0,33 \\
Ervilha & $0,58 \pm 0,02$ & 0,73 \\
Espinafre & $1,33 \pm 0,30$ & 0,65 \\
Jiló & $1,30 \pm 0,10$ & 1,14 \\
Nabo & $0,65 \pm 0,02$ & 0,40 \\
Pimentão & $0,61 \pm 0,00$ & 0,40 \\
Quiabo & $1,10 \pm 0,10$ & 0,38 \\
Repolho & $0,87 \pm 0,12$ & 0,45 \\
Tomate & $1,07 \pm 0,11$ & 0,76 \\
Vagem & $0,73 \pm 0,06$ & 0,53 \\
& &
\end{tabular}

\section{4 - CONCLUSÕES}

A partir dos resultados, observa-se que para a maioria das amostras, o cobre se encontra sob a forma de, no mínimo, 6 espécies químicas distintas, a extraída em cada um dos extratores I, II, III, IV e V e a fração não extraída. Estudos que permitam a identificação dos compostos extraídos pelos diferentes extratos utilizados poderão ser de grande valia para avaliação da biodisponibilidade do cobre.

\section{5 - REFERÊNCIAS BIBLIOGRÁFICAS}

[1] ANDRADE, E.C.B.; ASSIS, A.S.; FERREIRA, F.N.; MELLO, V.S.; TAKASE, I. Determinação dos teores de cobre e zinco em frutas, ANAIS, XVIII CBCTA. ISBN 85-89123$1-4,2002$.

[2] ANDRADE, E.C.B.; BARROS, A.M.; ASSIS, A.S.; FERREIRA, F.N.; MELLO, V.S.; TAKASE, I. Avaliação dos teores de cobre e zinco em hortaliças C cruas e 
após processamento térmico em meio salino e aquoso, ANAIS, XVIII CBCTA, ISBN 85-89123-01-4, 2002.

[3] ANDRADE, E.C.B.; BARROS, A.M.; MAGALHÃES, A.C.P.; CASTRO, L.L.S.; TAKASE, I. Avaliação da biodisponibilidade de cobre e zinco em cereais crus e processados termicamente em meio aquoso e salino. Revista Brasileira de Nutrição Clínica, v. 17, n. 3, p. 79-82, 2002.

[4] ANDRADE, E.C.B.; DRADE, E.C.B.; BARROS, A.M.; MAGALHÃES, A.C.P.; CASTRO, L.L.S.; TAKASE, I. Avaliação dos teores de cobre e zinco em leguminosas cruas e após processamento térmico em meio salino e aquoso, ANAIS, XVIII CBCTA, ISBN 85-89123-01-4, 2002.

[5] AOAC, OFFICIAL METHODS OF ANALYSIS. Association of official analytical Chemists, $40^{\mathrm{a}}$ ed, USA, 1984.

[6] BARCELOUX, D.G. Copper. Journal of Toxicology Clinical Toxicology, v. 37, n. 2 p. 217-30, 1999.

[7] COZZOLINO, S.M.F. Biodisponibilidade de Minerais. Revista Nutrição. Campinas, p. 87-98, jul.-dez., 1997.

[8] FAIRWEATHER-TAIT, S.J. Bioavaibility of dietary minerals. Biochemical Society Transactions Colchester, v. 24, n. 3, p. 775-780, 1996.

[9] FRANCO, G. Tabela de Composição de Alimentos. 9a ed. São Paulo: Editora Atheneu, 1999.

[10] GALlARDO-LARA, F.; AZCON, M.; QUESADA, J.L.; POLO, A. Phytoavaibility and Extractability of Copper and Zinc in Calcareous Soil Amended with Composted Urban Wastes. Journal of Environmental Science and Health, B, v. 34, p. 1049-1064, 1999.

[11] GLEYZES, C.; TEllier, S.; ASTRUC, M. et al. Fractionation studies of trace elements in contaminated Soils and sediments: a review of sequential extraction procedures Trends in Analytical Chemistry, v. 21, n. 6-7, 2002.

[12] IPOLY, J.; BRUNORI, C.; CREMISINI, C.; FODOR, P.; MACALUSO, L.; MORABITO, R. Evaluation of performance of time saving extraction devices in the BCR-three-step sequential extraction procedure. Journal Environmtal Monitoring, aug, v. 4, n. 4, p. 541-8, 2002.

[13] MAHAN, L. KATHELEEN, KRAUSE, MARIE Alimentos, Nutrição \& Dietoterapia, São Paulo: Rocca, 9a ed., 1998.

[14] MILLER, J.; MILLER, J. Statistics and chemometrics for analytical chemistry, $4^{\text {th }}$ ed., Inglaterra, Prentice Hall, 2001.

[15] OMS, ORGANIZAÇÃO MUNDIAL DE SAÚDE. Elementos Traço na Nutrição e Saúde. São Paulo: Roca, 1998.
[16] ORnellas, L. H. Técnica Dietética Seleção e Preparo de Alimentos, $3^{\text {a }}$ ed, Rio de Janeiro: Julio C Reis Livraria, 1974.

[17] PÉREZ-CID, B., LAVILla, I., BENDICHO, C. Speeding up of three-stage sequential extraction method for metal speciation using focused ultrasound, Analytica Chimica Acta, n. 360, p. 35-41, 1998.

[18] QIAN, J.; WANG, Z.; SHAN, X.; TU, Q.; WEN, B.; CHEN, B. Evaluation of plant availability of soil trace metals by chemical Fractionation d multiple regression analysis, Environmental Pollution, v. 91, n. 3, p. 309-315, 1996.

[19] RAMOS, L.; Gonzales, M.T.; HERNANDEZ, L.M.; Sequential Extraction of Copper, Lead, Cadmium, and Zinc in Sediments from Ebro River [Spain]: Relationship with Levels Detected in Earthworms. Bulletin of Environmental Contamination and toxicology, v. 62, n. 3, p. 301-308, 1999.

[20] SANDSTORM, B. Micronutrientes interactions:effects on absorption and bioavaibility. The British Journal of Nutrition, May, 85 Suppl 2:S181-5, 2001

[21] SANZ-MEDEL, A. Trace element analytical speciation in biological systems: importance, challenges and trens. Spectrochimica Acta Part B, n. 53, p. 197-211, 1998.

[22] SCHEINOST, A.C.; KRETZSCHMAE, R.; PFISTER, S.; ROBERTS, D.R. Combine selective sequential extraction, X-ray absorption spectroscopy, and principal components analysis in soil. Environmental Science \& Techonology, dec, v. 1, n. 36, 2002.

[23] SCHRAMEL, O.; MiChAlKe, B.; KETTRUP, A. Study of the copper distribution in contaminated soils of hop fields by single and sequential extraction procedures. Science Total Environmental, dec, v. 18, n. 263, p. 11-22, 2000.

[24] SHIOWATANA, J.; TANTIDANAI, N.; NOOKABKAEW, S.; NACAPRICHA, D. A novel continuous flow sequential extraction procedure for metal speciation in solids. Journal of Environmtal Quality, jul-aug, n. $30 ; 2001$.

[25] VULKAN, R.; MINGELGRIN, U.; BEN-ASHER, J.; FRENKEL, H. Copper and zinc speciation in the solution of a soil-sludge mixture. Journal of Environmental Quality, v. 31, n. 1, p. 193-203, jan.-feb., 2002.

[26] WAITZBERG, D.L. Nutrição Oral, Enteral e Parenteral na Prática Clínica. $3^{\text {a }}$ ed., São Paulo: Editora Atheneu, p. 131-134, 2002. 